D'une communauté de praticiens à un programme de recherche. Réflexions sur le développement de la pédagogie universitaire en Suisse romande

Shifting from a community of practitioners to a research programme. Considerations on the development of research in teaching and learning in higher education in French-speaking Switzerland

De una comunidad de prácticos a un programa de investigación. Reflexiones sobre el desarrollo de la pedagogía universitaria en Suiza romana

Nicole Rege Colet

Volume 34, numéro 3, 2008

La recherche sur la pédagogie de l'enseignement supérieur. Où en sommes-nous?

URI : https://id.erudit.org/iderudit/029511ar

DOI : https://doi.org/10.7202/029511ar

Aller au sommaire du numéro

Éditeur(s)

Revue des sciences de l'éducation

ISSN

0318-479X (imprimé)

1705-0065 (numérique)

Découvrir la revue

Citer cet article

Rege Colet, N. (2008). D'une communauté de praticiens à un programme de recherche. Réflexions sur le développement de la pédagogie universitaire en Suisse romande. Revue des sciences de l'éducation, 34(3), 623-641.

https://doi.org/10.7202/029511ar
Résumé de l'article

La Suisse romande se caractérise par une recherche en pédagogie universitaire très modeste. Pour comprendre cette situation, cet article propose un examen des sciences de l'éducation et de ses principaux axes de recherche dans les universités francophones. Il démontre l'absence d'un programme de recherche pris en charge par les structures universitaires alors que les centres de soutien à l'enseignement des universités mènent une recherche de terrain de qualité. L'essor des centres a donné lieu à une communauté de praticiens qui représentent désormais un fort potentiel de développement. En conclusion, la consolidation de la recherche en pédagogie universitaire passe par un renforcement de la collaboration entre les centres de soutien et les équipes académiques de recherche. 


\title{
D'une communauté de praticiens à un programme de recherche. Réflexions sur le développement de la pédagogie universitaire en Suisse romande
}

\author{
Nicole Rege Colet, professeure \\ Université de Lausanne
}

\begin{abstract}
RÉSUMÉ - La Suisse romande se caractérise par une recherche en pédagogie universitaire très modeste. Pour comprendre cette situation, cet article propose un examen des sciences de l'éducation et de ses principaux axes de recherche dans les universités francophones. Il démontre l'absence d'un programme de recherche pris en charge par les structures universitaires alors que les centres de soutien à l'enseignement des universités mènent une recherche de terrain de qualité. L'essor des centres a donné lieu à une communauté de praticiens qui représentent désormais un fort potentiel de développement. En conclusion, la consolidation de la recherche en pédagogie universitaire passe par un renforcement de la collaboration entre les centres de soutien et les équipes académiques de recherche.
\end{abstract}

MOTS CLÉS - recherche universitaire, tradition scientifique, structures institutionnelles, développement pédagogique, recherche appliquée.

\section{Introduction}

La Suisse romande se distingue par son histoire pédagogique, dont les premières avancées remontent au $18^{\mathrm{e}}$ siècle. L'héritage comprend d'illustres pédagogues dont les figures les plus marquantes sont Jean-Jacques Rousseau, Johann Pestalozzi ou, plus proche de nous, Jean Piaget. À sa manière, chacun a profondément marqué le paysage pédagogique romand et contribué au développement de l'expérimentation pédagogique puis de la recherche en éducation. Si d'aventure, le précepteur du jeune Émile, personnage central de l'œuvre de Rousseau (1966), venait à réapparaitre, il pourrait se féliciter de voir appliquer les principes pédagogiques qu'il utilisait pour apprendre à Émile à s'orienter dans la forêt de Montmorency. Basés sur l'expérimentation et le raisonnement inductif, ces principes sont plus connus, aujourd'hui, sous le terme d'apprentissage par problèmes. Piaget, pour sa part, pourrait s'enorgueillir d'entendre les enseignants revendiquer leur adhésion au socioconstructivisme et argumenter que la connaissance se construit par accumulation et accommodation. Sans aucun doute, l'héritage pédagogique est conséquent.

Outre sa riche tradition pédagogique, la Suisse romande se caractérise par une haute densité d'institutions d'enseignement supérieur, puisqu'elle accueille quatre 
universités de recherche, une école polytechnique fédérale et quatre hautes écoles spécialisées qui correspondent à des universités de sciences appliquées. En tout, ce sont plus de 153000 étudiants qui suivent une formation de niveau tertiaire pour un bassin de population totalisant 1800000 individus (Office fédéral de la statistique, 2004).

Avec un tel patrimoine et un foisonnement d'établissements de formation supérieure, on serait en droit d'attendre que la pédagogie universitaire occupe une place prépondérante dans le paysage helvétique de la recherche en éducation. Or, les premières observations ne confirment pas cette attente et dévoilent, bien au contraire, un paysage plutôt dénudé. Autant l'annoncer d'emblée, la recherche en pédagogie universitaire ne fait pas partie des fleurons de la recherche en éducation en Suisse romande. Il n'en demeure pas moins que l'idée même de la pédagogie universitaire connaît un développement important depuis dix ans, notamment avec la mise en place des services de soutien à l'enseignement. Il existe ainsi une communauté de praticiens à l'œuvre, qui travaillent le plus souvent en réseaux.

L'objectif de cet article est de faire comprendre la place actuelle de la recherche en pédagogie universitaire. Nous argumenterons, entre autres, sur le fait que la communauté des praticiens, certes centrée aujourd'hui sur les mesures d'accompagnement, pourrait être à l'origine d'un nouveau programme de recherche en pédagogie universitaire, puisque le terreau suisse est suffisamment fertile pour accueillir de nouvelles perspectives. Pour étayer cet argument, nous commencerons par esquisser le paysage scientifique de la recherche en éducation en Suisse romande, afin de situer les traditions scientifiques et la place des sciences de l'éducation. Nous présenterons, ensuite, les domaines d'études de la pédagogie universitaire, tels qu'ils se manifestent aujourd'hui dans les centres de recherche et les services de soutien à l'enseignement. Nous discuterons des conditions qui favorisent le passage d'un programme d'action à un programme de recherche, ce qui nous amènera à analyser de nouvelles formes de collaborations entre chercheurs et professionnels dans le domaine de l'enseignement universitaire.

\section{Le terrain d'étude : la Suisse romande et ses universités}

Notre examen de la recherche helvétique en pédagogie universitaire se cantonne à la Suisse romande, soit la partie francophone de la Suisse. Deux raisons dictent cette délimitation: la première tient au choix de ce numéro thématique qui vise un état de la situation dans plusieurs régions du monde, dont les pays francophones européens. La seconde tient aux spécificités culturelles et politiques de la Suisse. En effet, les différentes régions linguistiques (francophone, germanophone et italophone) se présentent comme autant d'environnements culturels, chacun se démarquant par ses références et ses réseaux de collaboration. La Suisse romande trouve ses alliances naturelles avec la France ou la Belgique, alors que la Suisse alémanique est plus proche de l'Allemagne et de l'Autriche. À cela, il faut ajouter que la souveraineté des 23 cantons de la Confédération helvétique permet à chaque 
canton de disposer de son propre appareillage politique, dont un système éducatif cantonal. Ainsi, la Suisse ne possède pas un système éducatif national, mais se présente comme une constellation de 23 systèmes qui s'étend jusqu'à la formation supérieure. Compte tenu de la diversité culturelle et de la multitude des réalités politiques et institutionnelles, il paraissait plus raisonnable de se limiter à la région francophone pour parler de la recherche en pédagogie universitaire.

Les quelques données factuelles qui suivent ont pour simple objectif de brosser le paysage universitaire de la Suisse romande. Le tableau est sciemment réduit au strict minimum pour éclairer le sujet et nous admettons qu'aux yeux de certains, il paraittra sans doute un peu réducteur. Le système d'enseignement supérieur suisse est un système dual qui distingue, d'une part, les institutions traditionnelles de recherche et de formation scientifique comprenant les universités et les écoles polytechniques et, d'autre part, les institutions de recherche appliquée et de formation professionnelle regroupées au sein des hautes écoles spécialisées (State Secretariat for Education and Research, 2005). Les universités sont toutes autonomes et cantonales, se référant aux autorités politiques de leur canton et, sur le plan fédéral, à une instance de coordination nationale, la Conférence universitaire suisse. Dans tous les domaines disciplinaires, les universités offrent des formations de base et avancée dans la recherche, et chaque établissement définit ses pôles d'excellence. Les écoles polytechniques ainsi que les hautes écoles spécialisées sont gérées sur le plan fédéral. Les hautes écoles spécialisées sont de création récente (au cours des années 1990), afin d'offrir une formation professionnelle de niveau tertiaire, qui complète la formation scientifique dispensée dans les universités et écoles polytechniques.

La région Suisse romande compte quatre universités (Fribourg, Genève, Lausanne et Neuchâtel) et une école polytechnique fédérale, à Lausanne. Les hautes écoles spécialisées de la région rassemblent plus d'une vingtaine d'établissements qui offrent des formations dans le domaine de la gestion, des arts appliqués, de l'ingénierie, de la santé, du social et de l'hôtellerie. Parmi ces hautes écoles, on trouve encore quatre établissements consacrés à la formation des enseignants du primaire et parfois du secondaire, soit les hautes écoles pédagogiques. Notre étude se concentre uniquement sur les universités et l'école polytechnique de Lausanne, car ce sont ces institutions qui abritent la recherche en éducation et les balbutiements de la recherche en pédagogie universitaire. La Suisse romande accueille également d'autres instituts de recherche en éducation (Institut de recherche et de documentation pédagogique, 2005), notamment avec les services cantonaux de recherche en éducation, présents dans les cantons de Genève, Vaud, Valais et du Jura ou encore, sur le plan régional, l'Institut de recherche et de documentation pédagogique à Neuchâtel et, finalement, une institution intercantonale, le Centre suisse de coordination de la recherche en éducation. Or, les domaines d'action de ces centres concernent le plus souvent le niveau de l'enseignement primaire ou secondaire, ou encore les premières étapes de la formation professionnelle. Ainsi, 
si nous voulons décrire la recherche en pédagogie universitaire en Suisse, c'est bien à l'intérieur des universités que nous devons poursuivre notre enquête.

\section{La recherche en pédagogie universitaire, premier état de la question}

Les sciences de l'éducation figurent en bonne place dans les universités de Suisse romande, même si leur insertion universitaire et institutionnelle varie d'un établissement à un autre. L'école polytechnique, qui a pour vocation la recherche et la formation dans les sciences de l'ingénierie et de la vie, n'est pas a priori l'établissement où l'on imagine trouver le plus fort développement. Nous verrons cependant que c'est là qu'ont eu lieu les premières tentatives d'institutionnalisation de la pédagogie universitaire. La structure la plus importante est, sans conteste, la Section des sciences de l'éducation au sein de la Faculté de psychologie et des sciences de l'éducation de l'Université de Genève. Elle comprend 10 groupes de recherches, animés par 14 professeurs et 22 collaborateurs de recherche (Section des sciences de l'éducation, 2004). Son positionnement institutionnel, en tant que structure indépendante au sein d'une faculté, lui donne une bonne visibilité et elle jouit d'une position unique en Suisse romande. Comme le relèvent les travaux rassemblés par Hofstetter et Schneuwly (1998b), elle jouit d'une expertise largement reconnue qui en fait une référence incontournable dans la région. Dans les autres universités, les sciences de l'éducation occupent une place plus réduite et connaissent des formes institutionnelles différentes: l'Université de Fribourg accueille un département en Faculté des lettres, l'Université de Lausanne abrite un Institut des sciences sociales et pédagogiques au sein de la Faculté des sciences sociales et politiques et la Faculté des lettres et sciences humaines de l'Université de Neuchâtel comprend un Institut des sciences de l'éducation. La dotation professorale de ces trois dernières structures n'est en rien comparable à celle de la Section de l'Université de Genève. Les équipes se composent d'un, voire de deux postes professoraux, avec un maximum de huit collaborateurs de recherche.

Les traditions scientifiques de recherche en éducation s'organisent autour de trois grands axes. Si plusieurs domaines y sont développés, peu touchent à la problématique de l'enseignement supérieur et encore moins à celle de la pédagogie universitaire. Les plus présentes sur la scène romande sont en lien avec les situations scolaires dans l'enseignement primaire et secondaire, qui constitue le premier axe. L'examen des programmes de recherches des équipes (Département des sciences de l'éducation, 2004; Section des sciences de l'éducation, 2004) indique un intérêt marqué pour les didactiques des disciplines, les processus d'apprentissage et d'évaluation en situation scolaire, la motivation à l'école et dans la formation professionnelle ainsi que les approches interculturelles de l'éducation. Le deuxième axe regroupe les équipes qui étudient des problématiques en lien avec les systèmes de formation, dont les politiques de gestion et d'administration de la formation, les politiques de qualité, l'innovation dans le système éducatif ou l'analyse des contextes et systèmes de formation (École polytechnique fédérale de Lausanne, 
2003; Unité Politique, Économie, Gestion et Éducation comparée, 2004). Finalement, le troisième axe comprend les recherches qui portent sur l'usage des technologies de l'information et de la communication (TIC) dans l'enseignement: multimédia et apprentissages, interaction homme-machine, apprentissage collaboratif et formation à distance (Technologie de la formation et de l'apprentissage, 2005). Ce type de recherche est plus particulièrement localisé dans l'unité de recherche spécialisée dans la technologie de la formation et de l'apprentissage de l'Université de Genève (TECFA). Ici, le processus d'apprentissage reste au cœur des recherches, mais il est éclairé par les apports des technologies éducatives.

\section{Comment qualifier la recherche en pédagogie universitaire?}

Au terme de cette énumération, il est important de préciser ce que recouvre la recherche en pédagogie universitaire. Selon notre acception, elle prend pour objet d'études l'enseignement supérieur et ses pratiques d'enseignement. Le questionnement porte, à notre sens, sur les spécificités de l'enseignement et de l'apprentissage en milieu universitaire (Biggs, 1999; Hativa, 2000; Langevin et Bruneau, 2000). Deux catégories d'acteurs sont concernées avec, d'une part, les étudiants à travers leur expérience universitaire, dont celle du métier d'étudiant et, d'autre part, les chercheurs-enseignants en rapport avec leur pratique enseignante. Les conditions qui favorisent l'apprentissage constituent le pôle apprenant de la recherche en pédagogie universitaire qui explore le profil des étudiants (Bédard et Viau, 2001; Frenay, Noël, Parmentier et Romainville, 1998), leur acclimatation à la vie universitaire (Coulon, 1997), les mesures de soutien aux étudiants et la réussite universitaire (Galley et Droz, 1999; Romainville, 2000). Le comment bien enseigner pour bien apprendre est au cour des études d'un second pôle, le pôle enseignant qui porte sur la planification curriculaire et pédagogique (Prosser et Trigwell, 1999; Ramsden, 1992). Il regroupe les travaux sur les réformes curriculaires et le degré de la cohérence des pratiques pédagogiques par rapport à ces réformes (Beaty, 2003), les pratiques pédagogiques et évaluatives innovatrices (Brown et Glasner, 1999; Edwards et Knight, 1995; Rege Colet et Romainville, 2006; Romainville, 2002), l'apport des technologies éducatives (Lebrun, 1999, 2002, 2005), les conceptions des enseignants sur l'enseignement et leurs modèles pédagogiques (Hativa et Goodyear, 2002; Kember et Kwan, 2002), l'organisation du travail des enseignants et le soutien pédagogique aux enseignants (Dejean, 2002; Donnay et Romainville, 1996; Knight, 2002; Trowler, 1998; Webb, 1996).

\section{Premier constat, l'absence de recherche institutionnelle}

Par pudeur ou par crainte d'une démarche trop introspective, les pratiques d'enseignement de l'université se dérobent comme objet d'étude. La pudeur est une excuse facile pour expliquer le peu d'enthousiasme; en réalité, l'explication est plus simple et tient aux mécanismes de financement de la recherche. Il n'existe en Suisse aucune structure de soutien pour la recherche en pédagogie universitaire, 
de telle sorte que ni la recherche financée par les universités ni celle soutenue par le Fonds national suisse n'ont cherché à mieux appréhender la pratique enseignante à l'Université. Ce déficit est illustré notamment dans le Programme national de recherche Efficacité des systèmes de formation (PNR 33), qui a été mené de 1993 à 1998. Sur les 39 projets sélectionnés, aucun ne traite de l'enseignement universitaire. Le rapport final (Berthoud, Hutmacher, Trier et Wachter, 1999) déplore l'absence d'une réflexion sur le niveau tertiaire et recommande d'orienter les recherches dans cette direction.

Outre le manque de financement, la pédagogie universitaire souffre d'un déficit d'image. Le plus souvent, elle n'est pas considérée comme un champ d'études à part entière, et les universités peinent à investir dans le domaine des sciences de l'éducation qui connaissent des problèmes de reconnaissance scientifique (Hofstetter et Schneuwly, 1998a). Cette difficulté a été accentuée par l'émergence des hautes écoles pédagogiques où se pose la question des différentes formes de recherche en éducation. La pédagogie universitaire se trouve prise dans un débat qui divise, d'une part, les protagonistes d'un programme de recherche en éducation et, d'autre part, les avocats de la mise en œuvre d'un programme d'action. Par delà les querelles d'écoles, la pédagogie universitaire se trouve régulièrement reléguée au statut de programme d'application pratique sous forme de soutien à l'enseignement.

Ce constat un peu amer, qui conclut à la non-prise en charge de la pédagogie universitaire par les sciences de l'éducation, à l'absence d'institutionnalisation d'une recherche programmatique, au manque de financement et au manque de crédibilité scientifique, décrit une image morose du paysage romand. Fort heureusement, il existe des exceptions et des nouveaux développements institutionnels qui laissent apercevoir des perspectives plus réjouissantes.

\section{L'École polytechnique, pionnière dans le domaine}

En fondant, en 1973, sa Chaire de Pédagogie et didactique, l'École polytechnique fédérale de Lausanne a fait œuvre de pionnière (Bagnoud et Suisse Bänziger, 1999). Elle a été la première haute école à nommer un professeur dont le cahier des charges couvrait explicitement le domaine de l'enseignement supérieur. Dans le prolongement des travaux de Perry (1970) sur l'expérience étudiante, les recherches menées pendant les 30 ans de l'existence de la chaire se sont plutôt orientées vers l'étude des pratiques d'études. Elles fournissent un excellent exemple du programme de recherche décrit par Alava et Romainville (2001) dans leur revue des traditions de recherche sur les études en milieu universitaire. La liste des publications témoigne d'une riche activité de recherche, avec une focalisation sur la cognition et l'apprentissage ou encore sur les méthodes d'enseignement ainsi que sur la réussite professionnelle des diplômés.

La Chaire de pédagogie et didactique est restée fidèle au domaine des pratiques d'études. Au cours des années 1990, l'École polytechnique a mis en place des ser- 
vices de soutien à l'enseignement, dont l'Unité d'évaluation de l'enseignement et d'insertion professionnelle (UNEED'IP) et divers services de soutien audio-visuel et technique. L'UNEED'IP a été la première structure à offrir un accompagnement pédagogique aux enseignants par le biais de l'évaluation formative de leurs enseignements et des activités de formation à l'enseignement en milieu universitaire.

À la fin des années 1990, la configuration de l’École polytechnique révèle une séparation institutionnelle entre, d'une part, une recherche traditionnelle dans le domaine des pratiques d'études assurée par la Chaire et, d'autre part, des activités de terrain conduites par un service administratif. En 2001, lors du départ à la retraite du titulaire de la Chaire, la direction de l'École a décidé de regrouper en une seule structure les missions de recherche et de services. C'est ainsi qu'est né, en 2002, le Centre de recherche et d'appui pour la formation et ses technologies (CRAFT), placé sous la direction d'un professeur d'université. L'équipe du CRAFT comprend aujourd'hui 15 personnes avec des conseillers pédagogiques, des chercheurs et du personnel administratif et technique.

En raison de l'expertise de l'actuel directeur du Centre, les recherches menées à l'École polytechnique ont complètement changé d'orientation pour privilégier le domaine du e-learning. Le rapport d'activités (CRAFT, 2005) fait état de trois thèmes de travail développés par l'axe Recherche: le travail collaboratif médiatisé, les technologies mobiles et la géolocalisation. Le paradigme de travail est résolument expérimentaliste, trouvant ses origines dans la psychologie expérimentale. Les résultats permettent d'éclairer la complexité des mécanismes d'apprentissage caractéristiques de la collaboration, mais ne prennent pas en compte la spécificité de l'enseignement universitaire. L'axe Formation, conseil, évaluation et enquête se trouve plus proche de la problématique, avec ses évaluations de différents programmes et formations, ses enquêtes sur les besoins de formation et son étude des pratiques d'évaluation des acquis des étudiants à l'École polytechnique.

Cette cohabitation des deux axes du CRAFT illustre parfaitement le partage usuel du territoire où un programme de recherche fondamentale, assuré par des chercheurs, côtoie un programme d'actions, pris en charge par des conseillers scientifiques dont les contours scientifiques sont plus flous. Mariage de raison ou alliance constructive, chaque regroupement connaît des issues plus ou moins heureuses.

\section{4. À l'Université de Fribourg, une chaire en didactique universitaire}

Les efforts consentis par l'École polytechnique ont largement contribué à l'essor d'une réflexion sur l'enseignement universitaire, mais la palme d'or pour la création d'une chaire de didactique universitaire revient, sans conteste, à l'Université de Fribourg, avec la première nomination professorale dans le domaine en 2002. Le Centre de didactique universitaire et nouvelles technologies et enseignement a pris sa forme actuelle en 2002, avec la création d'un pôle spécifique en pédagogie universitaire, qui s'ajoute aux activités centrées sur l'usage des nouvelles technologies dans l'enseignement supérieur (Centre de didactique universitaire et 
nouvelles technologies et enseignement, 2005). La direction du centre a été confiée à une professeure qui bénéficie d'une double insertion institutionnelle, puisqu'elle se réferre à la fois au département des sciences de l'éducation, pour la recherche, et au vice-recteur de l'enseignement, pour les activités de services.

Globalement, le Centre se concentre sur la formation des adultes et les technologies de l'éducation. Les projets en cours portent sur l'apprentissage chez les adultes et sur l'évaluation des dispositifs de formation innovants avec la participation à deux projets européens: EQUEL (e-Quality in e-Learning) et un projet portant sur la construction identitaire chez l'adulte. Nous retrouvons ici une centration de la recherche sur la pratique d'études et les conditions qui favorisent l'apprentissage, les questions traitant du métier de chercheur-enseignant ne sont pas encore abordées.

Sous l'angle de l'enseignement, la pédagogie universitaire est abordée dans un programme postdiplôme, did@cTIC formation en enseignement supérieur et nouvelles technologies de l'éducation. Ce cursus modulaire permet d'acquérir une formation de base dans la préparation et la conduite de dispositifs de formation. Les compétences développées concernent la planification pédagogique, les méthodes d'enseignement et d'apprentissage, la maîtrise des technologies éducatives, l'évaluation des apprentissages et de l'enseignement ainsi que la gestion du travail universitaire. Les mémoires de fin d'études fournissent des occasions de questionner la pratique enseignante et le métier de chercheur-enseignant.

Notre survol du paysage de la recherche en éducation en Suisse romande se conclut sur une présence réduite de la recherche en pédagogie dans les structures universitaires. Les premiers efforts observés se focalisent sur le pôle apprenant et se préoccupent moins du pôle enseignant. Ce bilan mitigé s'explique par la faible inscription institutionnelle de la recherche sur l'enseignement supérieur et une difficulté à positionner le domaine de la pédagogie universitaire dans les sciences de l'éducation. Les études dans le domaine ne sont pas valorisées sur le plan institutionnel ni sur le plan scientifique, et elles souffrent de non-reconnaissance scientifique.

\section{Deuxième constat, l'émergence des services de soutien}

L'examen des thèmes de recherche indique que l'analyse des pratiques enseignantes à l'université se situe plutôt dans les services de soutien à l'enseignement. Si la recherche a été délaissée sur le plan institutionnel, les établissements ont néanmoins investi dans les structures de soutien à l'enseignement. Comme les enseignants du supérieur sont le seul ordre à ne pas bénéficier d'une formation formelle en pédagogie, les universités ont dû faire face à une pression toujours croissante pour développer une offre de formation de qualité. En tant qu'institutions publiques, elles ont également été invitées à rendre compte de la manière dont elles remplissaient leurs missions de recherche et d'enseignement et à argumenter des procédures d'évaluation de la qualité répondant aux critères internationaux (Renaut, 2002). C'est donc dans un contexte de défense de l'autonomie et de la liberté universitaire 
que les services de soutien à l'enseignement ont commencé à voir le jour à la fin des années 1990. Rattachés directement aux directions des établissements et conçus comme des unités de services et de prestations aux enseignants, ces services se sont vu confier le mandat de valoriser les activités d'enseignement, de renforcer la qualité de l'enseignement et de la formation universitaire et de promouvoir les innovations éducatives et pédagogiques. Les programmes d'activités se sont rapidement modelés sur les axes de travail habituellement associés à ce type de centre (Kreber, 1997; Weimer, 1990; Wright, 1995), à savoir la formation du corps enseignant, le conseil pédagogique et l'évaluation formative de l'enseignement.

Les hautes écoles romandes ont opté pour des structures non universitaires (Rege Colet, 2006), détachées des facultés, qui recrutent des professionnels dans le domaine de l'enseignement supérieur. Différents rapprochements avec le milieu universitaire ont été pensés, par exemple en confiant la direction à un professeur ou en chapeautant les activités par une commission composée de représentants universitaires. Dans cette configuration, les centres de soutien à l'enseignement s'organisent autour d'activités de services, mais peuvent, dans certaines conditions, développer des activités de recherche.

Toutes les hautes écoles romandes disposent maintenant d'un centre structuré et bien implanté dans le paysage institutionnel. Le CRAFT à l'École polytechnique fédérale ainsi que le Centre de didactique universitaire et nouvelles technologies et enseignement à l'Université de Fribourg, tous deux mentionnés plus haut, sont complétés par le centre Formation et Évaluation de l'Université de Genève (FormEv), le Centre de soutien de l'enseignement (CSE) de l'Université de Lausanne et le Bureau du conseiller à l'enseignement de l'Université de Neuchâtel. Ces centres se reconnaissent dans leurs activités de terrain avec un premier axe consacré à la formation des enseignants, un deuxième axe dédié à l'évaluation de l'enseignement et des innovations pédagogiques, un troisième axe de conseil qui comporte plusieurs mesures d'accompagnement pédagogique et un quatrième axe plus spécifiquement orienté vers le développement du e-learning. Parmi les collaborateurs scientifiques qui y travaillent, il faut distinguer les généralistes, qui orientent leur travail sur le renforcement pédagogique des activités d'enseignement, et les spécialistes, qui se concentrent sur le e-learning. Le regroupement de ces deux profils relève d'un développement récent, car au début de la mise en place des centres, il était courant de séparer l'unité responsable de la pédagogie universitaire de celle qui se consacrait aux technologies éducatives avec, parfois, des difficultés à coordonner les activités.

Le tableau ne serait pas complet sans mentionner les services spécifiques des facultés de médecine. En effet, les deux facultés de médecine de Genève et de Lausanne disposent de leur propre unité de recherche en éducation médicale. Il s'agit, à Genève, de l'Unité de recherche et de développement en éducation médicale (UDREM) et, à Lausanne, de l'Unité de pédagogie médicale. Ces deux unités se sont spécialisées dans la recherche et la formation en pédagogie médicale, une 
sous-discipline de la pédagogie universitaire, avec son propre réseau de recherche et de publications scientifiques.

Au bout de presque 10 années d'activités dans les services de soutien, le paysage universitaire romand présente une solide communauté de praticiens qui se reconnaissent dans leur travail de promotion de l'enseignement supérieur et dans une identité professionnelle commune. Bien que les contours de cette nouvelle profession soient encore en cours de définition, les membres de cette communauté partagent les mêmes représentations quant au champ professionnel (principes et valeurs qui président au travail) et se retrouvent dans les actions proposées dans leurs établissements respectifs (Rege Colet, 2006). La communauté romande existe à travers des réseaux de collaboration qui ont pris forme: le Réseau romand de Conseil, Formation et Évaluation pour l'enseignement universitaire (www.rcfe.ch), qui réunit les collaborateurs des universités de Genève, de Lausanne et de Neuchâtel ainsi que de l'École polytechnique fédérale; le Réseau Girafe, ou Groupe interuniversitaire romand d'accompagnement et de formation en e-learning ( $h t t p: / /$ girafe.unige.ch/), spécialisé dans le e-learning; ou encore le Swiss faculty development network ( $h t t p: / / w w w . s f d n . u n i g e . c h /)$, une association nationale qui accueille tous les professionnels des universités et écoles polytechniques suisses.

La mission première des conseillers pédagogiques reste de l'ordre du service et comprend l'accompagnement des enseignants et équipes pédagogiques dans leurs initiatives pédagogiques, l'organisation d'activités de formation en pédagogie et l'évaluation de dispositifs pédagogiques variés. Cette mission de service est clairement dissociée d'une mission de recherche, et certains conseillers pédagogiques défendent leur orientation pratique. Généralement, les deux missions sont perçues comme antinomiques. Mais s'agit-il d'une incompatibilité intellectuelle? Ne faut-il pas plutôt parler en termes de positionnement professionnel et institutionnel? Les qualifications scientifiques de la plupart des conseillers pédagogiques en font des chercheurs, mais leur insertion institutionnelle, qui n'est pas universitaire, ne leur attribue pas explicitement une fonction de recherche. Or, des positions non universitaires, comme celle de collaborateur scientifique, ne devraient pas a priori exclure des activités de recherche. Le clivage est davantage dans l'esprit des gens, et certaines personnes ne s'autorisent pas cette activité de recherche ou ne reconnaissent pas leur participation à la recherche en éducation.

Les programmes d'activités des services de soutien à l'enseignement se sont bien étoffés au cours des dernières années. Or, ce développement s'accompagne d'un souci constant de documenter les actions menées, les résultats observés et l'impact sur le développement de la qualité de l'enseignement. Les enquêtes et mesures effectuées répondent aux mêmes critères que toute recherche en éducation, et les productions scientifiques qui en résultent trouvent une place honorable dans les publications du domaine. Dans l'impossibilité de proposer un inventaire exhaustif de ces dernières, nous nous contenterons d'indiquer les domaines qui ont fait l'objet d'une exploration systématique et détaillée. 


\section{Au service des usagers: une recherche de proximité, une recherche de terrain}

Sur le pôle apprenant, nous relevons les recherches menées sur la condition étudiante et la réussite universitaire. L'Université de Genève propose régulièrement des études sur la transition entre le secondaire et l'université (Maurice, 2001) et procède à des enquêtes à grande échelle sur les caractéristiques des étudiants et leur parcours universitaire (Atzamba et Petroff-Bartholdi, 2003; Schultheis et Stassen, 2005). Des études de même nature sur le changement d'orientation et l'abandon ont également été réalisées à l'Observatoire Science, Politique et Société de l'École polytechnique de Lausanne (Galley, Crettaz von Roten, et Alvarez, 2002).

Sur le pôle enseignant, à l'exception des travaux qui portent sur le e-learning, trois thèmes sont actuellement à l'étude dans les services de soutien: 1) l'impact de la formation dispensée aux enseignants; 2) les démarches d'évaluation et d'assurance de la qualité interne; et 3 ) la gestion des réformes curriculaires.

Les programmes de formation à l'enseignement universitaire sont offerts depuis plusieurs années. Il est donc normal d'en mesurer l'impact sur les bénéficiaires et l'effet sur la pratique enseignante. Lanarès (2004) a entrepris d'examiner les attentes et représentations des enseignants par rapport à la formation. Son analyse basée sur les réponses apportées à des questionnaires d'évaluation montre que les enseignants sont sensibles à des dimensions attribuées souvent aux adultes en formation des adultes (l'interactivité, la qualité des échanges) et à d'autres plus associées aux étudiants (la structure et l'organisation de la formation). De leur côté, Rege Colet et Barras (2004) ont développé un instrument d'évaluation de la performance enseignante lors d'une situation d'exposé frontal. Ils ont pu documenter le fait qu'une formation d'une semaine suivie d'un coaching sur le terrain permet des améliorations significatives dans les habiletés de communication.

Les travaux autour de l'évaluation concernent autant les pratiques en matière d'évaluation des apprentissages des étudiants que l'analyse des procédures d'évaluation de la qualité de l'enseignement. L'enquête sur l'évaluation des acquis (Ricci, 2004, 2006) menée à l'École polytechnique fédérale témoigne d'une démarche minutieuse d'analyse des conditions et résultats d'examen. Il s'agit d'un cas unique, voire exemplaire, de recherche systématique qui met en évidence une grande variété de pratiques chez les enseignants et documente les problèmes de validité et de fiabilité dans la manière d'apprécier les prestations des étudiants.

L'Université de Genève dispose d'un service d'aide à l'évaluation de l'enseignement, auquel les enseignants peuvent faire appel pour organiser l'évaluation de leurs enseignements. Depuis sa mise en place, ce service procède régulièrement à des enquêtes pour connaître le degré de satisfaction des usagers par rapport aux prestations offertes (Rege Colet, 2002) et pour mesurer l'impact de l'évaluation sur l'enseignement (Rege Colet et Durand, 2005). Les premiers résultats indiquent que, une fois les résistances initiales écartées, les enseignants commencent à s'approprier progressivement les outils de l'évaluation auprès des étudiants. Ce n'est 
que dans un second temps qu'ils peuvent exploiter les résultats pour réguler leurs enseignements. Il en résulte qu'une attitude positive à l'égard des pratiques d'évaluation procède d'un long processus d'adaptation et de changement culturel.

Le troisième domaine de questionnement concerne la gestion des réformes curriculaires. En effet, les universités suisses se sont engagées dans une réforme de leur offre de formation à la suite de la Déclaration de Bologne. De fait, les services de soutien ont été très impliqués dans ce processus, autant pour la réflexion sur l'avenir de la formation universitaire que pour les phases opérationnelles de renouvellement de l'enseignement. Dès lors, les enjeux liés à la planification curriculaire sont devenus une nouvelle manière d'aborder la pédagogie universitaire. Ce travail de transformation pédagogique représente un terrain d'études passionnant et, en tant qu'observateurs privilégiés des changements, les services de soutien ont commencé à proposer des analyses circonstanciées de l'impact des grandes réformes curriculaires sur les pratiques d'enseignement et la valorisation des activités d'enseignement (Durand, 2004; Rege Colet, 2004; Rege Colet et Durand, 2004).

La recherche sur le e-learning reste très présente dans les services de soutien, d'autant plus quand ils ont une mission explicite par rapport au développement des TIC. L'investissement se mesure par la participation aux projets européens qui visent à soutenir l'implantation des TIC dans l'enseignement, à former les enseignants et étudiants aux technologies éducatives et à mettre en place des réseaux de collaboration interuniversitaire pour mutualiser et exploiter le matériel pédagogique (Centre de didactique universitaire et nouvelles technologies de l'enseignement, 2005; Technologie de la formation et de l'apprentissage, 2005). Ces grands projets transversaux sont complétés par des enquêtes locales, où il s'agit de mieux comprendre le rapport des enseignants aux technologies éducatives dans leur travail afin d'orienter les services de soutien. Par exemple, l'Université de Genève a mené une enquête auprès de l'ensemble des enseignants sur leur utilisation des ressources électroniques et informatiques dans leur enseignement (Rege Colet, Bullat-Kolliker, Barras, et Lenzo Marchese, 2005).

Pour conclure ce survol des recherches, il convient de mentionner les travaux spécifiques des unités pédagogiques des facultés de médecine. La liste des publications de l'Unité de recherche en éducation médicale (2005), par exemple, fait état de nombreuses recherches sur l'apprentissage chez les étudiants et sur l'évaluation des compétences en lien avec des situations d'apprentissage innovantes. Leurs travaux s'intéressent aussi à la formation continue qui a été offerte au corps enseignant pour les préparer à leurs nouvelles fonctions enseignantes. Des travaux similaires sont menés par l'Unité de Pédagogie médicale de l’Université de Lausanne.

\section{Quel avenir pour la recherche en pédagogie universitaire en Suisse romande?}

Notre questionnement sur le statut de la recherche en pédagogie universitaire dans les universités romandes révèle une image quelque peu éclatée. Peut-il en être 
autrement? Est-ce vraiment une spécificité de la recherche en pédagogie ou n'est-ce pas une caractéristique de tout domaine de recherche que d'être composite? Nous avons pu montrer que les lieux de la recherche sont multiples et, parmi ceux-ci, que certains ne dépendent pas d'une mission explicite de recherche. Il existe donc une recherche formelle qui répond aux exigences universitaires usuelles et une recherche que nous qualifierons d'informelle qui est conduite en marge des lieux officiels. Faut-il en conclure à l'existence de deux catégories de recherche dont l'une serait supérieure à l'autre? À y regarder de plus près, nous ne discernons pas de différences épistémologiques ou méthodologiques fondamentales entre ces deux courants de la recherche. Tous les deux se rapportent aux mêmes références conceptuelles et théoriques. Les méthodes de travail de mesure et d'évaluation sont quasi identiques. Les publications et communications scientifiques sont soumises aux mêmes règles d'arbitrage.

Dans sa critique de la recherche, Kuhn (1983) postule que le paradigme de la recherche normale s'organise autour d'une théorie ou cadre conceptuel et d'une communauté scientifique. Il émet même l'hypothèse que l'existence de la communauté peut primer sur la présence d'une théorie constitutive. Au vu de la situation actuelle en Suisse romande, il est difficile de prouver l'existence d'un paradigme de recherche en pédagogie universitaire, d'une part parce que nous n'avons pas identifié un cadre référentiel susceptible d'agir en tant que fondement épistémologique et, d'autre part, parce que la communauté œuvrant dans le domaine se trouve divisée entre les chercheurs attitrés et les praticiens du terrain.

Selon nous, l'articulation des différentes activités d'interrogation de la pédagogie universitaire pourra se faire en fonction d'un projet commun. Pour atteindre un tel objectif, les acteurs du champ doivent relever plusieurs défis. Le premier défi consiste à évoluer d'un programme d'action de soutien à l'enseignement universitaire à un véritable programme de recherche. Il faut que ce dernier puisse bénéficier d'un soutien financier comme n'importe quel autre programme de recherche. Pour cela, il importe d'obtenir une reconnaissance formelle et scientifique de la pédagogie universitaire comme domaine d'études faisant partie intégrante de la recherche en éducation. Cela signifie ensuite que les institutions universitaires s'engagent à investir dans la recherche sur l'enseignement universitaire et à reconnaître que l'Université peut devenir objet d'études. Une telle prise en compte de la pratique enseignante universitaire procède d'un changement culturel, voire d'une révolution scientifique, pour reprendre une idée chère à Kuhn. Les résistances à l'égard de cette idée restent nombreuses et expliquent pourquoi la constitution d'un programme de recherche en pédagogie universitaire représente un défi, d'autant plus quand la recherche en éducation est perçue comme peu crédible, et celle sur la pédagogie universitaire, comme un bricolage.

Le deuxième défi consiste à organiser la communauté de manière à faire cohabiter des enseignants-chercheurs universitaires traditionnels et des praticiens du terrain. Le rapprochement doit être plus qu'un mariage de raison et se fonder sur 
une reconnaissance mutuelle des compétences et des profils professionnels des uns et des autres. Or, l'esprit de caste qui prédomine en milieu universitaire rend difficiles ces rencontres. La communauté pourra prendre forme à travers des expériences répétées de travail en réseau et en expérimentant de nouvelles formes de collaborations entre les structures universitaires et les services de soutien. Le but de ces collaborations n'est pas d'imposer une tutelle universitaire aux praticiens du terrain. Il s'agit, bien au contraire, de faire converger des expertises différentes au service d'une meilleure compréhension de l'enseignement universitaire. Ces collaborations doivent pouvoir s'ancrer dans des projets concrets où les contributions des uns et des autres sont clairement posées et où la parité entre partenaires est reconnue. L'argument d'Hofstetter et Schneuwly (1998a) est que cette complémentarité entre chercheurs et professionnels relève d'une spécificité des sciences de l'éducation qui doit être admise comme telle.

D'aucuns diront que ces objectifs sont ambitieux et prendront du temps à atteindre. Or, le renouvellement actuel de la formation supérieure en Suisse fournit un contexte général plutôt favorable. En effet, les enjeux politiques et économiques qui bousculent l'enseignement supérieur et les nouvelles contraintes auxquelles doivent faire face les institutions obligent un nouvel examen des missions d'enseignement et de formation, notamment la manière dont les activités d'enseignement sont prises en compte. Il n'est pas exclu que les politiques de formation supérieure traduisent enfin cette nécessité d'investir dans une recherche de qualité et en appellent aux compétences existantes. La question de la qualité de la formation supérieure figure désormais en bonne place dans l'agenda politique et peut, dès lors, prétendre à des mesures de soutien.

\section{Conclusion}

Notre réflexion sur la pédagogie universitaire en Suisse romande nous permet de conclure que la recherche se trouve en gestation. Au cours des dernières années, elle a certes été fécondée par des apports multiples et on peut observer un développement embryonnaire. La période de gestation et de maturation se poursuit et devrait naturellement aboutir à la naissance d'un véritable programme de recherche. Nous avons apporté l'argument qu'un projet commun pourrait aider à rassembler les éléments qui composent l'actuel paysage.

En 2005, la tenue, en Suisse romande, du $22^{\mathrm{e}}$ congrès de l'Association internationale de pédagogie universitaire (AIPU) a joué un rôle important pour rassembler les forces et donner une nouvelle orientation à la recherche. La nature même du congrès, centré sur la pédagogie universitaire, a donné une bonne visibilité institutionnelle et scientifique à la problématique. La participation active conjointe des différentes sections romandes des sciences de l'éducation ainsi que des services de soutien confirme l'intérêt que chercheurs et praticiens manifestent à l'égard du développement de l'enseignement universitaire. L'organisation du congrès, qui associe autant les chercheurs que les professionnels, a ainsi signé un rapproche- 
ment. Et finalement, les communications et conférences faites par des chercheurs confirmés et des praticiens expérimentés ont témoigné du double apport à la recherche en pédagogie universitaire et du dialogue possible. Il est donc permis de croire que ce congrès a donné un nouvel élan à la recherche en pédagogie universitaire en Suisse romande.

ENGLISH TITLE - Shifting from a community of practitioners to a research programme. Considerations on the development of research in teaching and learning in higher education in French-speaking Switzerland

SUMmARY - Research in higher education pedagogy is little developed in French-speaking Switzerland. In order to understand this, this article reviews the position of educational sciences and the associated research traditions within the universities. It underscores the lack of a research programme in the field within the traditional academic structures whereas faculty development units carry out sound research in higher education. The growth of these units has produced a community of practice with a strong potential for research. The article concludes that the development of research in higher education requires strengthening the collaboration between the faculty development units and the academic research teams.

KEY WORDS - university research, scientific tradition, institutional structures, faculty pedagogical development, applied research.

TíTULO EN ESPAÑOL • De una comunidad de prácticos a un programa de investigación. Reflexiones sobre el desarrollo de la pedagogía universitaria en Suiza romana

RESUMEN • La Suiza romana se caracteriza por una investigación muy modesta en pedagogía universitaria. Para entender esta situación, este artículo propone un examen de las ciencias de la educación y de sus principales ejes de investigación en las universidades francófonas. Demuestra la ausencia de un programa de investigación a cargo de las estructuras universitarias mientras los centros de apoyo a la enseñanza de las universidades llevan una investigación de campo de calidad. Los avances de los centros han dado lugar a una comunidad de prácticos que representan, de ahora en adelante, un fuerte potencial de desarrollo. En conclusión, la consolidación de la investigación en pedagogía universitaria conlleva un refuerzo de la colaboración entre los centros de apoyo y los equipos académicos de investigación.

PALABRAS CLAVES - investigación universitaria, tradición científica, estructuras institucionales, desarrollo pedagógico, investigación aplicada.

\section{Références}

Alava, S. et Romainville, M. (2001). Les pratiques d'étude, entre socialisation et cognition. Revue française de pédagogie, 136, 159-180.

Atzamba, H. et Petroff-Bartholdi, C. (2003). Étudiants 2001. Genève, Suisse: Université de Genève.

Bagnoud, K. et Suisse Bänziger, N. (1999). La Chaire de pédagogie et didactique fête ses 25 ans et publie son curriculum vitce. Ecublens, Suisse: École polytechnique fédérale de Lausanne. 
Beaty, L. (2003). The Bologna Process - an English perspective. Texte présenté au Bologna Process International Seminar. Bruxelles: Belgique.

Bédard, D. et Viau, R. (2001). Le profil d'apprentissage des étudiantes et étudiants de l'Université de Sherbrooke. Sherbrooke, Québec: Université de Sherbrooke.

Berthoud, A.-C., Hutmacher, W., Trier, U. P. et Wachter, T. (1999). La formation quel apport? Résultats des recherches du Programme national de recherche "Efficacité de nos systèmes de formation» PNR 33. Zurich, Suisse: Éditions Ruegger.

Biggs, J. (1999). Teaching for quality learning at University. Buckingham, United Kingdom: Society for Research into Higher Education and Open University.

Brown, S. et Glasner, A. (1999). Assessment Matters in Higher Education. London, United Kingdom: Society for Research into Higher Education and Open University.

Centre de didactique universitaire et nouvelles technologies et enseignement (2005). Formation did@cTIC. [En ligne].Disponible le 14 novembre 2005: www.unifr.ch/didactic/ article.php3?id_article $=21$

Coulon, A. (1997). Le métier d'étudiant. L'entrée dans la vie universitaire. Paris, France: Presses universitaires de France.

Centre de recherche et d'appui pour la formation et ses technologies (CRAFT) (2005). Rapport d'activités 2004. Lausanne, Suisse: École polytechnique fédérale de Lausanne.

Dejean, J. (2002). L'évaluation de l'enseignement dans les universités françaises. Paris, France: Haut Conseil pour l'évaluation de l'école.

Département des sciences de l'éducation (2004). Programme de recherche du fonds national motivation. [En ligne]. Disponible le 8 mars 2005: http://www.unifr.ch/ipg/sitecrt/ RechercheProjets/Motivation/Motivation.htm

Donnay, J. et Romainville, M. (1996). Enseigner à l'université: un métier qui s'apprend? Bruxelles, Belgique: De Boeck.

Durand, N. (2004). De la gestion de projet au projet pédagogique - exemple de la réforme de Bologne à l'Université de Genève. Actes du $21^{\mathrm{e}}$ congrès de l'AIPU. Marrakech, Maroc: Association internationale de pédagogie universitaire.

Edwards, A. et Knight, P. (1995). Assessing competence in higher education. London, United Kingdom: Kogan Page.

École polytechnique fédérale de Lausanne (2003). Travaux et publications de l'Observatoire science, politique et société. [En ligne]. Disponible le 8 mars 2005: http://osps.epfl.ch/FR/ Recherche/publications.html

Frenay, M., Noël, B., Parmentier, P. et Romainville, M. (1998). L'étudiant-apprenant. Bruxelles, Belgique: De Boeck.

Galley, F., Crettaz von Roten, F. et Alvarez, E. (2002). Changement d'orientation et abandon des études en $1^{\mathrm{re}}$ année à l'EPFL: rapport de la $1^{\mathrm{re}}$ étape, volée 2000-2001. Ecublens, Suisse: Observatoire science, politique et société.

Galley, F. et Droz, R. (1999). L’abandon des études universitaires. Des difficultés du métier d'étudiant. Fribourg, Allemagne: Éditions universitaires de Fribourg.

Hativa, N. (2000). Teaching for effective learning in Higher Education. Dordrecht, Holland: Kluwer Academic Publishers. 
Hativa, N. et Goodyear, P. (2002). Teacher thinking, beliefs and knowledge in Higher Education. Dordrecht, Holland: Kluwer Academic Publishers.

Hofstetter, R. et Schneuwly, B. (1998a). Sciences de l'éducation entre champs disciplinaires et champs professionnels. Dans R. Hofstetter et B. Schneuwly (Dir.) : Le pari des sciences de l'éducation. Bruxelles, Belgique: De Boeck.

Hofstetter, R. et Schneuwly, B. (1998b). Le pari des sciences de l'éducation. Bruxelles, Belgique: De Boeck.

Institut de recherche et de documentation pédagogique (2005). Institut de recherche et de documentation pédagogique: centres de recherche. [En ligne]. Disponible le 8 mars 2005: http://148.196.1.118/scripts/irdp/actions/lc/view-centre.pl

Kember, D. et Kwan, K. (2002). Lecturers' approaches to teaching and their relationship to conceptions of good teaching. Dans N. Hativa et P. Goodyear (Dir.): Teacher Thinking, Beliefs and knowledge in Higher Education. Dordrecht, Holland: Kluwer Academic Publishers.

Knight, P. (2002). A systemic approach to professional development: learning as practice. Teaching and teacher education, 18(3), 23-41.

Kreber, C. (1997). Evaluation of instructional development programs in Ontario Universities. Higher education perspectives, 1(1), 23-41.

Kuhn, T. S. (1983). La structure des révolutions scientifiques. Paris, France: Flammarion.

Lanarès, J. (2004, mai). Se former à la pédagogie universitaire: quelles sont les représentations et les attentes des enseignants. Actes du $21^{\mathrm{e}}$ congrès de l'AIPU. Marrakech, Maroc: Association internationale de pédagogie universitaire.

Langevin, L. et Bruneau, M. (2000). Enseignement supérieur. Vers un nouveau scénario. Issyles-Moulineaux, France: ESF éditeur.

Lebrun, M. (1999). Des technologies pour enseigner et apprendre. Bruxelles, Belgique: De Boeck.

Lebrun, M. (2002). Théories et modèles pédagogiques pour enseigner et apprendre. Quelle place pour les TIC dans l'éducation? Bruxelles, Belgique: De Boeck.

Lebrun, M. (2005). e-Learning pour enseigner et apprendre. Allier pédagogie et technologie. Louvain-la-Neuve, Belgique: Academia-Bruylant.

Maurice, D. (2001). Réussir la première année à l'université. La transition secondaire-université: le projet Boussole. Revue française de pédagogie, 136, 77-86.

Office fédéral de la statistique (2004). Degrés d'éducation, chiffres clés. [En ligne]. Disponible le 10 mars 2005: http://www.bfs.admin.ch/bfs/portal/fr/index/themen/bildung_und_wissenschaft/bildung/bildungsstufen/kennzahlen0/hochschule_r/uebersicht.html

Perry, W. (1970). Forms of intellectual and ethical development. New York, New York: Holt, Rinehart and Winston.

Prosser, M. et Trigwell, K. (1999). Understanding learning and teaching: the experience in higher education. Buckingham, United Kingdom: Society for Research into Higher Education and Open University.

Ramsden, P. (1992). Learning to teach in higher education. London, United Kingdom: Routledge. 
Rege Colet, N. (2002). L'évaluation de l'enseignement par les étudiants. Phase de généralisation 1998-2001. Genève, Suisse: Université de Genève.

Rege Colet, N. (2004). Le processus de Bologne: de la réforme des curricula à la rénovation de l'enseignement. Actes du $21^{\mathrm{e}}$ congrès de l'AIPU. Marrakech, Maroc: Association internationale de pédagogie universitaire.

Rege Colet, N. (2006). Représentations et modèles pédagogiques des conseillers pédagogiques en milieu universitaire. Dans N. Rege Colet et M. Romainville (Dir.) : La pratique enseignante en mutation à l'université. Bruxelles, Belgique: De Boeck.

Rege Colet, N. et Barras, H. (2004). Préparer les assistants débutants à l'enseignement universitaire. Un programme de formation: principes et cadre de travail. Actes du $21^{\mathrm{e}}$ congrès de l'AIPU. Marrakech, Maroc: Association internationale de pédagogie universitaire.

Rege Colet, N., Bullat-Kolliker, C., Barras, H. et Lenzo Marchese, G. (2005). E-learning@ unige: les tendances générales. Genève, Suisse: Université de Genève.

Rege Colet, N. et Durand, N. (2004). Working on the Bologna Declaration: promoting integrated curriculum development and fostering conceptual change. International journal for academic development, 9(2), 167-170.

Rege Colet, N. et Durand, N. (2005). Évaluation de l'enseignement à l'Université de Genève. Mesures d'impact sur les pratiques enseignantes. Genève, Suisse: Université de Genève.

Rege Colet, N. et Romainville, M. (2006). La pratique enseignante en mutation à l'université. Bruxelles, Belgique: De Boeck.

Renaut, A. (2002). Que faire des universités? Paris, France: Bayard.

Ricci, J.-L. (2004). Quelle est la corrélation entre la satisfaction des étudiants quant aux examens et la note qu'ils ont reçue? Actes du $21^{\mathrm{e}}$ congrès de l'AIPU. Marrakech, Maroc: Association internationale de pédagogie universitaire.

Ricci, J.-L. (2006). L'évaluation des examens est-elle un sujet négligé de la pédagogie universitaire? Dans N. Rege Colet et M. Romainville (Dir.): La pratique enseignante en mutation à l'université. Bruxelles, Belgique: De Boeck.

Romainville, M. (2000). L'échec dans l'université de masse. Paris, France: L'Harmattan.

Romainville, M. (2002). L'évaluation des acquis dans l'enseignement universitaire. Paris, France: Haut conseil de l'évaluation de l'enseignement.

Rousseau, J.-J. (1966). Émile ou De l'éducation. Paris, France: Flammarion.

Schultheis, F. et Stassen, A. (2005). Étudiants 2004. Genève, Suisse: Université de Genève.

Section des sciences de l'éducation (SSED) (2004). Publications de la Section des sciences de l'éducation. [En ligne]. Disponible le 10 mars 2005: http://www.unige.ch/fapse/publications.html

State Secretariat for Education and Research (2005). The federalist education system in Switzerland. [En ligne]. Disponible le 14 novembre 2005: www.sbf.admin.ch/htm/ bildung/bildung-e.html

Technologie de la formation et de l'apprentissage (2005). Research at TECFA. [En ligne]. Disponible le 10 mars 2005: http://tecfa.unige.ch/tecfa/research/research-overview.html

Trowler, P. R. (1998). Academics responding to change. New higher education frameworks and academic cultures. Buckingham, United Kingdom: Society for Research into Higher Education and Open University. 
Unité de recherche en éducation médicale (UDREM) (2005). Recherche, projets et publications. [En ligne]. Disponible le 9 mars 2005: http://edumed.unige.ch/udrem/recherche/ recherche.html

Unité Politique, Économie, Gestion et Éducation comparée (2004). La recherche. [En ligne]. Disponible le 10 mars 2005: http://www.unige.ch/fapse/pegec/Recherche.htm

Webb, G. (1996). Understanding staff development. Buckingham, United Kingdom: Society for Research into Higher Education and Open University.

Weimer, M. (1990). Improving college training. San Francisco, California: Jossey Bass.

Wright, W. A. (1995). Teaching improvement practices: successful strategies for higher education. Bolton, Massachusetts: Anker Publishing.

$\mathrm{M}^{\text {me }}$ Rege Colet est professeure à la Haute École pédagogique de Lausanne après avoir exercé à l'Université de Genève.

\section{Correspondance}

nicole.rege-colet@hepl.ch

Ce texte a été révisé par Dominique Lafleur.

Texte reçu le: 5 octobre 2005

Version finale reçue le: 17 septembre 2007

Accepté le: 18 mars 2008 